\title{
Adición de coque de petróleo a mezclas coquizables. Modificación de la calidad del coque metalúrgico ${ }^{(\bullet)}$
}

\author{
J.A. Menéndez ${ }^{(*)}$, J.J. Pis ${ }^{(*)}$, M.A. Díez ${ }^{(*)}$, C. Barriocanal ${ }^{(*)}$, M.D. Casal $^{(*)}$, J.B. Parra $\left(^{(*)}\right.$ \\ y R. Álvarez ${ }^{(*)}$
}

Resumen Se estudia la influencia que la adición de un coque verde de petróleo a mezclas coquizables tiene sobre la calidad de los coques metalúrgicos resultantes. Se midieron propiedades mecánicas (ensayo IRSID), reactividad (ensayos CEE y NSC) y textura (porosidad y distribución del tamaño de poros). En general, la adición de coque de petróleo da lugar a una disminución de la porosidad de los coques obtenidos tanto a escala de laboratorio como semiindustrial. Dicha disminución se debe, en gran medida, a la disminución de microporos, lo cual contribuye a explicar la de la reactividad frente al $\mathrm{CO}_{2}$ de los coques metalúrgicos. Se desarrolla una metodología para la obtención y evaluación de las propiedades de coques metalúrgicos a escala de laboratorio, cuyos resultados se comparan con los obtenidos a escala semiindustrial.

Palabras clave: Coque de petróleo. Coque metalúrgico. Resistencia mecánica. Reactividad. Textura.

\section{Petroleum coke addition to coking coal blends. Modification of the quality of resultant metallurgical cokes}

\begin{abstract}
The effects of a green petroleum coke addition on coke metallurgical quality in terms of mechanical strength (IRSID test), reactivity to carbon dioxide (CEE and NSC tests) and texture (porosity and pore size distribution) are described. Petroleum coke addition, generally decreases porosity, both to semiindustrial and laboratory scale mainly due to the microporosity, which helps to explain the observed decrease in the reactivity to $\mathrm{CO}_{2}$. A laboratory-scale methodology to assess the main properties of metallurgical cokes was developed. Laboratory results were backed by tests to semiindustrial scale.
\end{abstract}

Keywords: Petroleum coke. Metallurgical coke. Mechanical strength. Reactivity. Texture.

\section{INTRODUCCIÓN}

El coque de petróleo se utiliza como aditivo a las mezclas destinadas a la producción de coques metalúrgicos por los fabricantes de coques de todo el mundo, desde el decenio de 1940. Sin embargo, este uso no se ha mantenido de forma continuada, sino que, dependiendo de los precios del coque de petróleo en comparación con el de los carbones coquizables con contenidos medios y bajos de volátiles, se

\footnotetext{
(•) Trabajo recibido el día 27 de enero de 1995.

(*) Instituto Nacional del Carbón, INCAR (CSIC). Aptdo. 73. 33080-Oviedo (España).
}

consideraba o desestimaba su utilización, según que dicho precio fuera más bajo o más elevado que el de estos últimos (1). En la actualidad, la tendencia de los precios, tanto del coque de petróleo combustible (abundante y barato) como de los carbones coquizables (más escasos y caros), hace que la industria productora de coque metalúrgico muestre, de nuevo, interés hacia este tipo de aditivo.

La utilización de coque de petróleo a lo largo de los años se ha realizado con bastante éxito, tanto en lo que respecta al ahorro económico como a la mejora de la calidad de los coques metalúrgicos obtenidos (2-4). En este sentido, uno de los factores a considerar es la cantidad óptima de coque de petróleo que se debe añadir. En cualquier caso, ha 
de tenerse en cuenta que el contenido de azufre del coque de petróleo va a ser un factor limitante de la cantidad a adicionar en el caso de los coques metalúrgicos, aunque en algunos países del Este de Europa se han utilizado grandes proporciones de coque de petróleo con alto contenido de azufre para la obtención de coques destinados a industrias metalúrgicas no férreas (5).

A pesar de que existen varios trabajos que estudian el efecto de la adición de coque de petróleo, especialmente en lo que se refiere al tamaño, morfología y orientación de las estructuras cristalinas de los coques obtenidos (textura óptica) mediante microscopía óptica de luz polarizada y a ciertas propiedades de microrresistencia mecánica (6-8), se hace necesario profundizar sobre el tema, especialmente en momentos como el actual en el que parece renacer el interés por la utilización del coque de petróleo en la industria productora de coque metalúrgico.

En el presente trabajo se estudia el efecto que la adición de diferentes cantidades de coque de petróleo tiene sobre las principales características (resistencia mecánica, reactividad y textura) de los coques metalúrgicos producidos tanto a escala de laboratorio como semiindustrial. Los resultados obtenidos son de aplicación directa a escala industrial.

\section{PARTE EXPERIMENTAL}

\subsection{Materiales}

A escala de laboratorio se utilizó una mezcla industrial de carbones, pasta B132, de las empleadas habitualmente por la Empresa Nacional Siderúrgica Española, S.A. (ENSIDESA) y un carbón individual, Welch, de bajo contenido de materia volátil y baja plasticidad. Con objeto de comparar los resultados obtenidos, la mezcla B132 se coquizó también a nivel semiindustrial. Las características, tanto del carbón individual como de la pasta, se muestran en la tabla I.

Los carbones anteriormente mencionados se coquizaron con diversas cantidades de un coque de petróleo, R1, suministrado por Repsol Derivados, S.A., cuyas características se muestran en la tabla I.

El coque de petróleo R1 es del tipo verde combustible, con un contenido elevado de azufre. Dicho coque de petróleo se mezcló, tanto con el carbón individual como con la mezcla industrial, en cantidades que variaron hasta un $20 \%$, para la realización de las carbonizaciones a escala de laboratorio. A escala semiindustrial, en la que únicamente se utilizó la mezcla B132, la proporción de coque de petróleo se limitó al $6 \%$ con el fin de no sobrepasar el porcentaje máximo admisible de azufre $(1 \%)$ en mezclas industriales.
TABLA I.- Características principales del carbón Welch, pasta B132 y coque de petróleo R1

TABLE I.- Main characteristics of Welch coal, B132 blend and petroleum coke $R I$

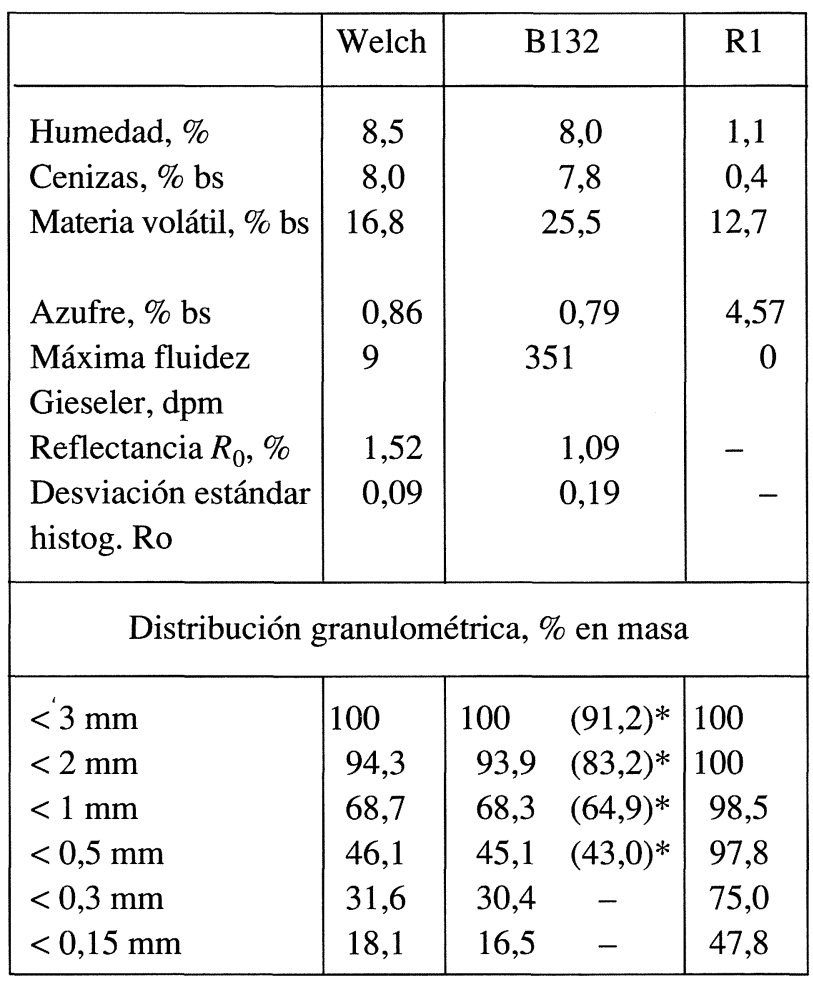

bs: calculado sobre base seca; dpm: divisiones por minuto; * Granulometría empleada a escala semiindustrial.

\subsection{Equipos y métodos}

A escala de laboratorio, las mezclas se carbonizaron en un horno con capacidad para $400 \mathrm{~g}$, con calentamiento eléctrico en la solera y con un dispositivo que permite la coquización a volumen constante. La densidad de carga empleada en todos los casos fue de $820 \mathrm{~kg} / \mathrm{m}^{3}$. Las muestras se coquizaron en atmósfera de nitrógeno, con una velocidad de calentamiento en la solera de $5 \mathrm{~K} / \mathrm{min}$, desde 800 hasta $1.200{ }^{\circ} \mathrm{C}$, manteniendo esta temperatura final hasta alcanzar $1.000{ }^{\circ} \mathrm{C}$ en la superficie superior de la carga de coque. El esquema de este horno se muestra en la figura 1.

A escala semiindustrial se utilizó el horno de $6 \mathrm{t}$ de capacidad de la Coquería Experimental del INCAR. Con anterioridad, se han publicado detalles referentes a esta planta (9). Los ensayos de coquización se realizaron a una densidad de carga de $710 \pm 5 \mathrm{~kg} / \mathrm{m}^{3}$, con una temperatura media de calentamiento en canales de alrededor de $1.250{ }^{\circ} \mathrm{C}$ y durante un tiempo total de coquización de $18 \mathrm{~h}$.

La resistencia mecánica de los coques obtenidos a escala de laboratorio se evaluó utilizando el método MITREM-INCAR (10 y 11), en el que partículas 


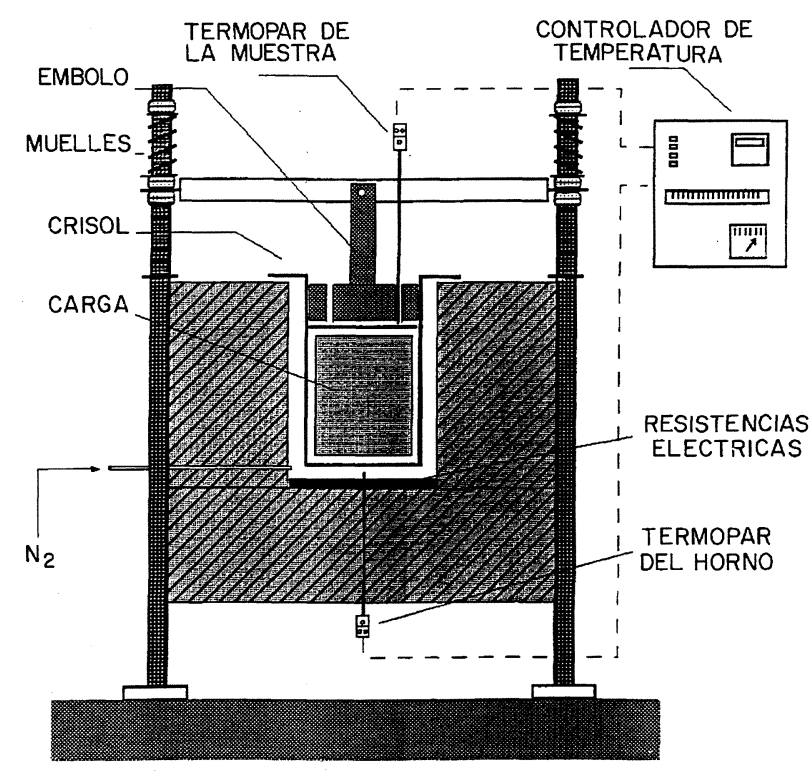

FIG. 1.- Esquema del dispositivo de coquización a escala de laboratorio (horno INCAR-H500).

FIG. 1.-Diagram of the furnace used in the laboratory carbonizations (INCAR-H500).

de coque de $5-15 \mathrm{~mm}$ se someten a un giro de 3.000 vueltas a una velocidad de $33 \mathrm{rpm}$ en un tambor cilíndrico de $110 \mathrm{~mm}$ de largo por $130 \mathrm{~mm}$ de diámetro. El índice de abrasividad se define como el tanto por ciento de material producido de tamaño inferior a $1 \mathrm{~mm}$.

En el caso de los coques obtenidos a escala semiindustrial, la resistencia mecánica se midió utilizando el ensayo IRSID (norma AFNOR M 03023), calculando los índices de cohesión $I_{20}$ (\% en masa $>20 \mathrm{~mm})$ y de abrasividad $I_{10}(\%$ en masa $<$ $10 \mathrm{~mm})$.
Para determinar la reactividad de los coques obtenidos, tanto a escala de laboratorio como semiindustrial, se utilizó el ensayo CEE-INCAR (12), y para los obtenidos a escala semiindustrial se empleó, además, el ensayo NSC (12 y 13).

La porosidad de los coques obtenidos se calculó a partir de la medida de las densidades real (helio) y aparente (mercurio) utilizando partículas de coque con un tamaño comprendido entre 1 y $3 \mathrm{~mm}$. Para los coques obtenidos a escala semiindustrial, la porosidad total se midió utilizando el método convencional descrito en la norma ASTM D 167-7, a partir de las densidades real y aparente determinadas por desplazamiento de agua. La distribución de volumen de poros se llevó a cabo con un porosímetro de mercurio Carlo Erba Macropore 2000, que permite obtener el volumen de macroporos con radios de poro comprendidos entre $7.500>r_{\mathrm{p}}>25$ $\mathrm{nm}$, y mesoporos, con radios comprendidos entre $25 \mathrm{~nm}>r_{\mathrm{p}}>3,7 \mathrm{~nm}$, calculándose el volumen de microporos $\left(r_{\mathrm{p}}<3,7 \mathrm{~nm}\right)$ por diferencia con respecto al volumen total de poros. Las isotermas de $\mathrm{CO}_{2}$ a $273 \mathrm{~K}$, a partir de las cuales se obtiene el volumen de microporos $\left(r_{\mathrm{p}}<2 \mathrm{~nm}\right)$, se obtuvieron con un equipo Micromeritics, ASAP 2000.

\section{RESULTADOS Y DISCUSIÓN}

La adición de coque de petróleo produce una disminución de las principales propiedades plásticas de las mezclas, tal y como se muestra en la tabla II, en la que se observa que tanto la máxima fluidez Gieseler (dpm) como el intervalo plástico $\left(T_{\mathrm{s}}-T_{\mathrm{r}}\right)$ disminuyen al aumentar el contenido de coque de petróleo en la mezcla, siendo $T_{\mathrm{s}}$ la temperatura de resolidificación y $T_{\mathrm{r}}$ la de reblandecimiento. Asimismo, se

TABLA II.- Evolución de la plasticidad Gieseler con la adición de coque de petróleo

TABLE II.- Influence of petroleum coke addition on Gieseler plasticity

\begin{tabular}{|l|c|c|c|c|c|c|c|c|c|c|c|}
\cline { 2 - 11 } \multicolumn{1}{c|}{} & \multicolumn{4}{|c|}{ Pasta B132, } & \multicolumn{6}{c|}{ Welch, } \\
\cline { 2 - 12 } & 0 & 3 & 6 & 10 & 20 & 0 & 2 & 4 & 6 & 10 & 20 \\
\hline$T_{\mathrm{r}},{ }^{\circ} \mathrm{C}$ & 411 & 412 & 413 & 412 & 413 & 446 & 445 & 449 & 446 & 450 & 454 \\
$T_{\mathrm{f}},{ }^{\circ} \mathrm{C}$ & 459 & 457 & 458 & 458 & 456 & 478 & 480 & 476 & 473 & 476 & 473 \\
$T_{\mathrm{S}},{ }^{\circ} \mathrm{C}$ & 494 & 492 & 493 & 490 & 487 & 500 & 501 & 497 & 497 & 494 & 492 \\
$T_{\mathrm{S}}-T_{\mathrm{r}},{ }^{\circ} \mathrm{C}$ & 83 & 80 & 80 & 78 & 74 & 54 & 56 & 48 & 51 & 44 & 38 \\
$F, \mathrm{dpm}$ & 351 & 219 & 152 & 115 & 37 & 9 & 7 & 5 & 4 & 3 & 2 \\
\hline
\end{tabular}

$T_{\mathrm{r}}$ : temperatura de reblandecimiento; $T_{\mathrm{f}}$ : temperatura de máxima fluidez; $T_{\mathrm{s}}$ : temperatura de resolidificación; $F$ : máximạ fluidez Gieseler; dpm: divisiones por minuto. 
observa una tendencia a la disminución de la temperatura de máxima fluidez $\left(T_{\mathrm{f}}\right)$ a medida que aumenta el contenido de coque de petróleo.

El desarrollo de la textura porosa de los coques metalúrgicos está condicionado, en gran medida, por los fenómenos que tienen lugar durante el intervalo plástico (14), que puede dividirse en tres etapas: una primera en la que la matriz carbonosa presenta elevada fluidez, los gases escapan fácilmente de la misma y apenas se desarrollan poros, ya que los huecos que provoca la salida de estos gases se rellenan con facilidad; una segunda etapa en la que aumenta la viscosidad y los huecos que dejan los gases son más difíciles de rellenar, dando lugar al desarrollo de grandes poros; por último, ya cerca de la temperatura de resolidificación, la gran viscosidad de la matriz evita el crecimiento de las burbujas y el tamaño de los poros es menor. Teniendo en cuenta esta relación entre plasticidad y desarrollo de la textura porosa, cualquier modificación de la primera traerá consigo variaciones en el desarrollo poroso de los coques resultantes, tal y como se pondrá de manifiesto a continuación.

\subsection{Textura porosa}

En las tablas III, IV y V se muestran los datos referentes a la textura porosa de los coques metalúrgicos obtenidos a partir de las mezclas de carbón Welch + R1 (Tabla III) y pasta B132 + R1 (Tabla IV), a escala de laboratorio, y pasta $\mathrm{B} 132+\mathrm{R} 1$, a escala semiindustrial (Tabla V).

TABla III.- Propiedades de textura de los coques obtenidos a escala de laboratorio. Welch + R1

TABLE III.- Textural properties of cokes obtained at laboratory scale. Welch $+R 1$

\begin{tabular}{|l|c|c|r|c|}
\cline { 2 - 5 } \multicolumn{1}{c|}{} & \multicolumn{4}{c|}{ Adición de coque de petróleo, \% } \\
\cline { 2 - 5 } \multicolumn{1}{c|}{} & \multicolumn{1}{c|}{0} & \multicolumn{1}{c|}{4} & \multicolumn{1}{c|}{10} & \multicolumn{1}{c|}{20} \\
\hline$d_{\mathrm{r}}, \mathrm{g} / \mathrm{cm}^{3}$ & 1,91 & 1,92 & 1,93 & 1,93 \\
$d_{\mathrm{a}}, \mathrm{g} / \mathrm{cm}^{3}$ & 1,50 & 1,52 & 1,57 & 1,60 \\
Porosidad, \% & 21,5 & 20,8 & 18,7 & 17,1 \\
$V_{\mathrm{p}}, \mathrm{mm}^{3} / \mathrm{g}$ & 143,1 & 137,1 & 118,8 & 106,9 \\
$V_{\text {macro }}, \mathrm{mm}^{3} / \mathrm{g}$ & 104,1 & 99,6 & 88,1 & 91,1 \\
$V_{\text {meso }}, \mathrm{mm}^{3} / \mathrm{g}$ & 6,5 & 7,2 & 4,4 & 3,7 \\
$V_{\text {micro }}, \mathrm{mm}^{3} / \mathrm{g}$ & 32,5 & 30,2 & 26,3 & 12,1 \\
$W_{\mathrm{o}}, \mathrm{mm}^{3} / \mathrm{g}$ & 5,8 & 4,9 & 3,1 & 2,5 \\
\hline
\end{tabular}

$d_{\mathrm{r}}$ : densidad real $(\mathrm{He}) ; d_{\mathrm{a}}:$ densidad aparente $(\mathrm{Hg})$; $V_{\mathrm{p}}$ : volumen total de poros; $V_{\text {macro }}, V_{\text {meso }}$ y $V_{\text {micro }}$ : calculados por porosimetría de $\mathrm{Hg} ; W_{\mathrm{o}}$ : volumen de microporos calculado por adsorción de $\mathrm{CO}_{2}$ a $273 \mathrm{~K}$.
TABla IV.- Propiedades de textura de coques obtenidos a escala de laboratorio. B132+ R1

TABLE IV.- Textural properties of cokes obtained at laboratory scale. $B 132+R 1$

\begin{tabular}{|l|r|r|c|c|c|}
\cline { 2 - 6 } \multicolumn{1}{c|}{} & \multicolumn{5}{|c|}{ Adición de coque de petróleo, \% } \\
\cline { 2 - 6 } \multicolumn{1}{c|}{} & \multicolumn{1}{c|}{0} & 3 & 6 & 10 & 20 \\
\hline$d_{\mathrm{r}}, \mathrm{g} / \mathrm{cm}^{3}$ & 1,91 & 1,91 & 1,92 & 1,92 & 1,92 \\
$d_{\mathrm{a}}, \mathrm{g} / \mathrm{cm}^{3}$ & 1,60 & 1,63 & 1,64 & 1,66 & 1,68 \\
Porosidad, \% & 16,2 & 14,7 & 14,6 & 13,5 & 12,2 \\
$V_{\mathrm{p}}, \mathrm{mm}^{3} / \mathrm{g}$ & 101,4 & 89,9 & 88,9 & 81,6 & 74,4 \\
$V_{\text {macro }}, \mathrm{mm}^{3} / \mathrm{g}$ & 72,6 & 72,0 & 70,5 & 73,2 & 64,6 \\
$V_{\text {meso }}, \mathrm{mm}^{3} / \mathrm{g}$ & 7,9 & 5,0 & 9,1 & 7,2 & 9,1 \\
$V_{\text {micro }}, \mathrm{mm}^{3} / \mathrm{g}$ & 20,9 & 12,9 & 9,3 & 1,2 & 0,7 \\
\hline
\end{tabular}

$d_{\mathrm{r}}$ : densidad real $(\mathrm{He}) ; d_{\mathrm{a}}:$ densidad aparente $(\mathrm{Hg})$; $V_{\mathrm{p}}$ : volumen total de poros; $V_{\text {macro }}, V_{\text {meso }}$ y $V_{\text {micro }}$ : determinados por porosimetría de $\mathrm{Hg}$.

TÁBla V.- Propiedades de textura de coques obtenidos a escala semiindustrial. B132+R1

TABLE V.- Textural properties of cokes obtained at semiindustrial scale. B132+RI

\begin{tabular}{|c|c|c|c|c|}
\hline & \multicolumn{4}{|c|}{ Adición de coque de petróleo, $\%$} \\
\hline & 0 & 2 & 4 & 6 \\
\hline$d_{\mathrm{r}}, \mathrm{g} / \mathrm{cm}^{3(1)}$ & 1,96 & 2,01 & 1,95 & 2,00 \\
\hline$d_{\mathrm{a}}, \mathrm{g} / \mathrm{cm}^{3}(1)$ & 0,92 & 0,95 & 0,96 & 0,99 \\
\hline Porosidad, \% (1) & 53,1 & 52,7 & 50,8 & 50,5 \\
\hline$d_{\mathrm{r}}, \mathrm{g} / \mathrm{cm}^{3(2)}$ & 1,91 & 1,92 & 1,92 & 1,92 \\
\hline$d_{\mathrm{a}}, \mathrm{g} / \mathrm{cm}^{3}(2)$ & 1,65 & 1,68 & 1,69 & 1,71 \\
\hline Porosidad, \% (2) & 13,6 & 12,5 & 12,0 & 11,2 \\
\hline$V_{\mathrm{p}}, \mathrm{mm}^{3} / \mathrm{g}(2)$ & 83,0 & 74,8 & 70,9 & 65,9 \\
\hline$V_{\text {macro }}, \mathrm{mm}^{3} / \mathrm{g}(3)$ & 55,1 & 53,3 & 53,8 & 54,9 \\
\hline$V_{\text {meso }}, \mathrm{mm}^{3} / \mathrm{g}(3)$ & 5,9 & 6,1 & 6,1 & 6,3 \\
\hline$V_{\text {micro }}, \mathrm{mm}^{3} / \mathrm{g}(3)$ & 22,0 & 15,4 & 11,0 & 4,5 \\
\hline
\end{tabular}

$d_{\mathrm{r}}$ : densidad real; $d_{\mathrm{a}}$ : densidad aparente; (1): determinadas por picnometría de agua según la norma ASTM D 167-7; (2): determinadas por medidas de densidad con $\mathrm{He}$ y $\mathrm{Hg}$; (3): determinados por porosimetría de $\mathrm{Hg}$.

En general, se observa que para todos los coques la densidad real $(\mathrm{He})$ experimenta un ligero aumento, mientras que la densidad aparente $(\mathrm{Hg})$ aumenta claramente con la adición de coque de petróleo a las mezclas. Esto trae como consecuencia una disminución de la porosidad y del volumen de poros con radio inferior a $7.500 \mathrm{~nm}$, tanto en los coques obtenidos en laboratorio (Tablas III y IV), como en los obtenidos a escala semiindustrial (Tabla V). En el 
caso de estos últimos, la medida de la porosidad total mediante métodos basados en picnometría de agua, según la norma ASTM D 167-7, produce valores de porosidad mucho mayores, puesto que, debido a los mayores tamaños de las muestras de coque $(>50$ $\mathrm{mm}$ ) y a las características intrínsecas del método, mide poros de tamaño muy superior a los $7.500 \mathrm{~nm}$ de radio. En concordancia con los resultados obtenidos anteriormente, la evaluación de la porosidad mediante este método conduce nuevamente a una disminución de la porosidad al aumentar el porcentaje de coque de petróleo en la mezcla inicial.

En lo que respecta a la distribución de tamaño de poros, se observa cómo, en general, el volumen de macroporos disminuye con el aumento del porcentaje de coque de petróleo. El volumen de mesoporos disminuye en el caso de los coques obtenidos a partir de las mezclas del carbón Welch, y permanece más o menos constante en el caso de los coques obtenidos a partir de las mezclas con la pasta B132. Por último, se observa una clara disminución del volumen de microporos a medida que aumenta la proporción de coque de petróleo. En el caso de los coques obtenidos con las mezclas de carbón Welch, se utilizó la técnica de adsorción de $\mathrm{CO}_{2}$ a $273 \mathrm{~K}$ para calcular el volumen de microporos menores de $2 \mathrm{~nm}$, a partir de la ecuación de Dubinin-Raduskevich (15), lo que corroboró la disminución del volumen de microporos puesta de manifiesto cuando se utilizó porosimetría de mercurio y medida de la densidad de helio.

El coque verde de petróleo no presenta propiedades plásticas, lo que contribuye a una disminución de la plasticidad de la mezcla. Sin embargo, no es un material totalmente inerte, ya que los volátiles que desprende durante la etapa plástica de la mezcla van a interaccionar con la matriz fluida, de tal forma que la aglomeración de las partículas de coque de petróleo será mejor y provocará menos fisuras en la interfase que si se tratase de un material totalmente inerte.

La adición de un material con bajo contenido de volátiles, como es el coque de petróleo, produce una disminución relativa del contenido de materia volátil de la masa a carbonizar, lo que unido al estrechamiento del intervalo plástico originado por la adición de coque de petróleo trae como consecuencia que se desprenda durante dicho intervalo plástico una menor proporción de materia volátil, responsable de la formación de poros.

Además, la adición de coque de petróleo provoca un aumento de la viscosidad de la mezcla durante el intervalo plástico, de tal forma que la velocidad de desprendimiento de la materia volátil es más lenta, produciéndose reacciones colaterales de una parte de dichos volátiles con la matriz plástica (16), lo que contribuye a una mayor densificación del coque metalúrgico resultante.
Los factores anteriormente mencionados explican cómo la adición de coque de petróleo da lugar a que disminuya la porosidad de los coques metalúrgicos resultantes, que se pone especialmente de manifiesto, según los datos obtenidos, en una reducción del volumen de microporos. En este sentido, cabe destacar que una parte de los microporos se produce una vez formado el semicoque, debido a los huecos originados por el desprendimiento de pequeñas moléculas de $\mathrm{CO}, \mathrm{CH}_{4}, \mathrm{H}_{2}, \mathrm{SH}_{2}$, etc. (16). Por tanto, el menor contenido de hidrógeno y heteroátomos en las mezclas, al adicionar un material como el coque de petróleo, generalmente con contenidos porcentuales de carbono superiores al de los carbones, contribuye a que la formación de la microporosidad por este mecanismo sea menor.

\subsection{Resistencia mecánica}

Para los coques obtenidos a partir de los ensayos realizados en el laboratorio, la resistencia mecánica se midió utilizando el ensayo de abrasividad MITREM-INCAR (11). En la figura 2 se representa la variación del índice de abrasividad con la cantidad de coque de petróleo añadido. Se observa cómo a medida que aumenta el porcentaje de coque de petróleo en las mezclas se obtienen coques metalúrgicos con menor índice de abrasividad. La explicación de este comportamiento podría atribuirse a la disminución observada de la porosidad.

Esta mejora en la calidad de los coques se corrobora con los resultados obtenidos a escala semiindustrial. Tal y como se observa en la figura 3, disminuye la abrasividad, medida en este caso como el índice $I_{10}$ del ensayo IRSID, y aumenta el índice de

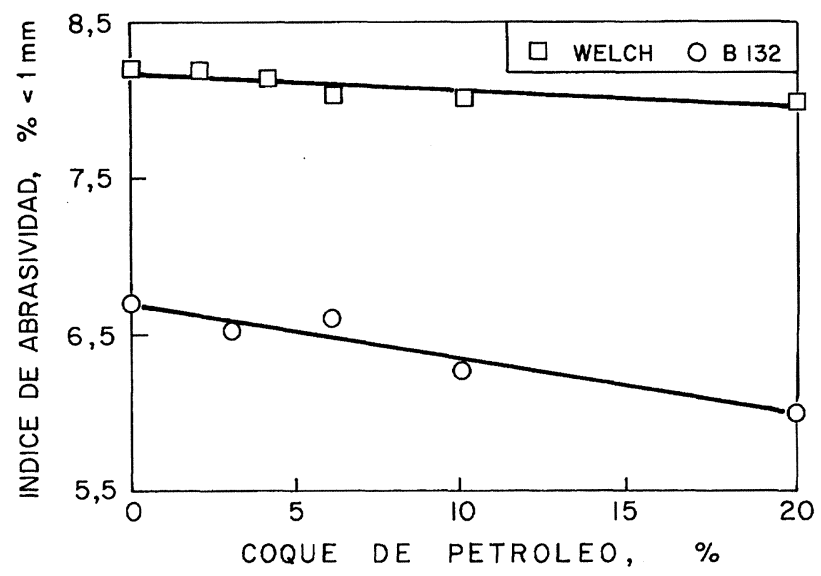

FIG. 2.- Variación del índice de abrasividad MITREM-INCAR con la adición de coque de petróleo.

FIG. 2.- Variation of the MITREM-INCAR abrasivity index with petroleum coke added. 


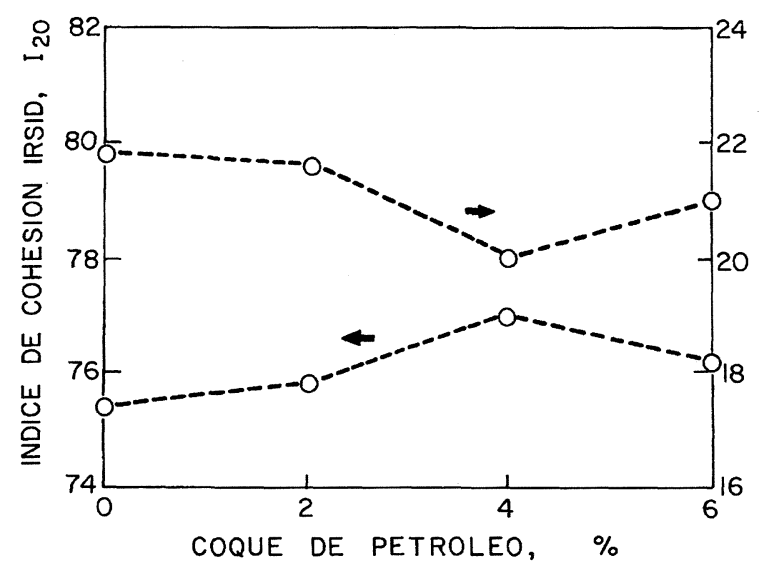

FIG. 3.- Variación de la resistencia mecánica (ensayo IRSID) con la adición de coque de petróleo.

FIG. 3.- Variation of mechanical strength (IRSID test) with petroleum coke added.

cohesión, $I_{20}$. No obstante, a diferencia de los resultados obtenidos a escala de laboratorio, se observa que los resultados óptimos de resistencia mecánica se consiguen con una adición del $4 \%$ de coque de petróleo. Esto se debe a que al aumentar la proporción de coque de petróleo que la matriz plástica tiene que aglomerar, los defectos de la unión entre el coque de petróleo y la matriz de coque metalúrgico empiezan a cobrar mayor importancia. Por otro lado, los mejores resultados obtenidos a escala de laboratorio para mezclas con porcentajes de coque de petróleo superiores al $4 \%$, se deben a las diferentes condiciones de coquización, fundamentalmente, a la mayor densidad de carga utilizada. Cabe esperar, por tanto, que la coquización en baterías industriales con hornos de mayor altura que los de la coquería experimental del INCAR pueda conducir a resultados más próximos a los de laboratorio.

\subsection{Reactividad frente al $\mathrm{CO}_{2}$}

La reactividad de los coques, obtenidos tanto a escala de laboratorio como semiindustrial, se evaluó utilizando el método CEE-INCAR (12); los resultados obtenidos se muestran en las figuras $4 \mathrm{y}$ 5 , respectivamente. En todos los casos, se observa una disminución de la reactividad al aumentar el porcentaje de coque de petróleo añadido.

Los coques obtenidos a escala semiindustrial se sometieron al ensayo de reactividad y resistencia mecánica después de reacción de la NSC (13), utilizado por la mayoría de las industrias siderúrgicas mundiales en el control de la calidad de los coques metalúrgicos que producen. En la figura 6 se representa la variación del índice de reactividad, CRI, y del índice de resistencia mecánica después de reacción, CSR, frente a la proporción de coque de

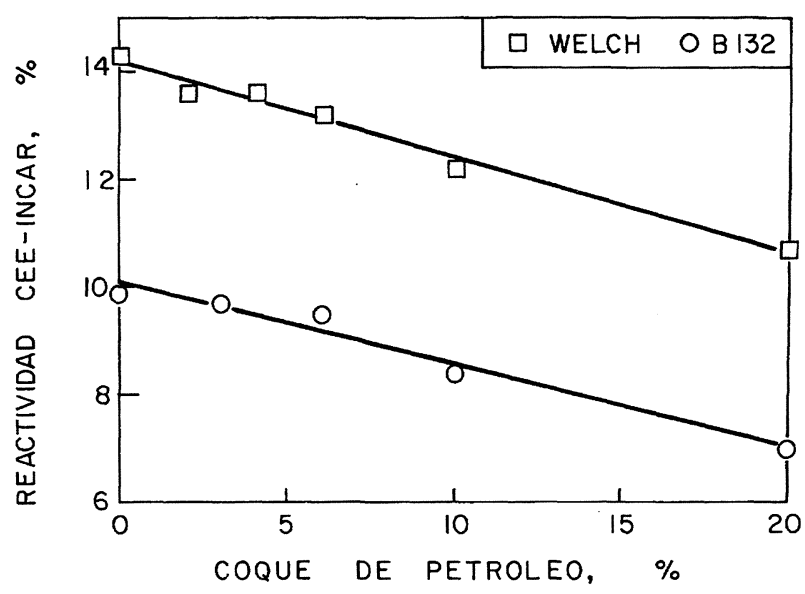

FIG. 4.- Variación de la reactividad CEE-INCAR con la adición de coque de petróleo, de los coques obtenidos a escala de laboratorio.

FIG. 4.-Variation of CEE-INCAR reactivity with petroleum coke added. Laboratory scale.

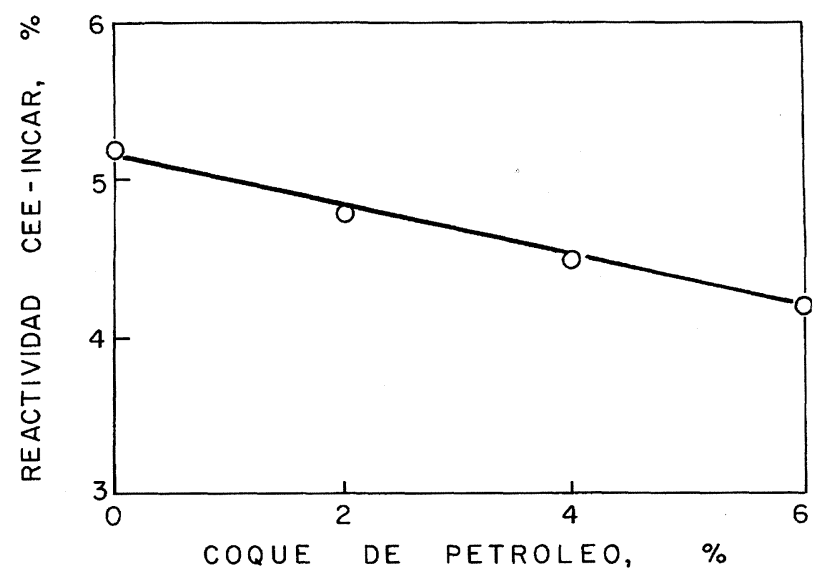

FIG. 5.- Variación de la reactividad CEE-INCAR con la adición de coque de petróleo, de los coques obtenidos a escala semiindustrial.

\section{FIG. 5.- Variation of CEE-INCAR reactivity with petroleum coke added. Semiindustrial scale.}

petróleo presente en las mezclas coquizadas. De nuevo se observa una disminución de la reactividad de los coques obtenidos y, dada la relación existente entre estos dos parámetros (12 y 13), un aumento de la resistencia mecánica después de la reacción.

Los factores a los que se puede atribuir la disminución que se observa en la reactividad de los coques metalúrgicos, al aumentar el porcentaje de coque de petróleo en las mezclas a coquizar, son:

- El coque de petróleo está formado por estructuras más ordenadas, del tipo "dominios" y "dominios fluidos", que son, por tanto, menos reactivas que las del coque metalúrgico (8). 


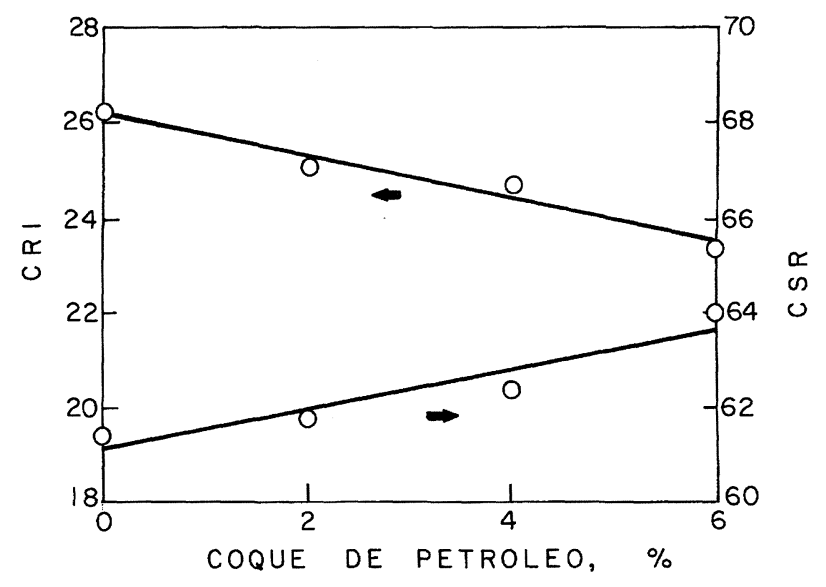

FIG. 6.- Variación de la reactividad (CRI) y resistencia mecánica después de reacción (CSR) con la adición de coque de petróleo, de los coques obtenidos a escala semiindustrial.

FIG. 6.-Variation of reactivity index, CRI and postreaction strength index, CSR with petroleum coke added. Semiindustrial scale.

- El coque de petróleo tiene un bajo contenido de cenizas, por lo que, al ser añadido a carbones coquizables, las mezclas resultantes tendrán menor contenido de las mismas, disminuyendo de esta manera el posible efecto catalítico de las cenizas en la reactividad de los coques resultantes (17).

- Disminuye el volumen de microporos, lo que contribuye a dificultar la difusión de los gases reactivos al interior de las partículas de coque metalúrgico.

La contribución de los dos primeros factores no parece suficiente para explicar la disminución observada de los valores de reactividad, puesto que los porcentajes de coque de petróleo añadidos no son muy elevados, especialmente para los coques metalúrgicos obtenidos a escala semiindustrial. Por lo tanto, la disminución del volumen de microporos parece ser el factor más importante.

\section{CONCLUSIONES}

La proporción del coque de petróleo en las mezclas está limitada a fin de no sobrepasar el contenido máximo admisible de azufre.

La adición de coque de petróleo a mezclas coquizables da lugar a una disminución de las propiedades plásticas de las mezclas, que a su vez con- tribuye a la disminución del volumen total de poros y especialmente del de microporos, de los coques metalúrgicos obtenidos. Dentro de los límites estudiados, la resistencia mecánica de los coques metalúrgicos aumenta con la adición de coque de petróleo mientras que su reactividad disminuye. Esta disminución de la reactividad está relacionada, principalmente, con la disminución del volumen de microporos. Los resultados obtenidos a nivel de laboratorio, mediante el sistema empleado, son extrapolables cualitativamente a escala industrial.

\section{Agradecimiento}

Los autores agradecen a REPSOL DERIVADOS, S.A. la financiación de este trabajo y a ENSIDESA el suministro de los carbones utilizados.

\section{REFERENCIAS}

(1) Lin, C. y Garcella, J. Ironmaking Proc. Metall. Soc. AIME , 44, 1985: 93-101.

(2) Triska, A.A. y Schubert, C.D. Congr. Intern. de Charleroi, C9, 1966.

(3) BCRA. Carbonization Res. Rep., 9, 1974.

(4) Valia, H.S. Ironmaking Proc. Metall. Soc. AIME, 91, 1992: 435-447.

(5) Koshkarov, V.Y., Danil'yan, P.G., VdovichenKo, N.S. Koshikarova, M.E. y Zhitnikov, P.N. Coke Chem. USSR, (trad. ing.), 3, 1982: 43-45.

(6) PAtrick, J.W. y Stacey, A.E. Fuel, 57, 1978: 258-264.

(7) Forrest, M.A. y MARSH, H. Fuel, 60, 1981: 429-433.

(8) Ruíz, O., Romero-Palazón, E., Díez, M.A. y Marsh, H. Fuel, 69, 1990: 456-458.

(9) Álvarez, R., Álvarez, E., Suárez, C., Méndez de Andés, F., Fernández, E. y SiRgado, M. 1st Int. Cokemaking Cong. Essen (R.F.A.), 1, 1987: E5.

(10) Pis, J.J., Cagigas, A., Simón, P. y LoRenzana, J.J. Fuel Proc. Technol., 20, 1988: 307-316.

(11) MEnÉndeZ, J.A. El coque de petróleo como aditivo en la producción de coques metalúrgicos. Tesis Doctoral. Universidad de Oviedo, 1994.

(12) Menéndez, J.A., Álvarez, R. y Pis, J.J. Rev. Metal. Madrid, 29 (4), 1993: 214-222.

(13) BCRA. Carbonization Res. Rep. 91, 1980.

(14) Koba, K., SaKata, K. e IdA, S. Fuel, 65, 1986: 307-311.

(15) Dubinin, M.M. Progress in Surface and Membranes Science. Vol. 9. Cadenhead, D. Ed. Academic Press. Nueva York, 1975: 1-70.

(16) Loison, R., Foch, P. y Boyer, A. Coke Quality and Production. Ed. Butterworths. Londres, 1989: 108-110.

(17) Goscinski, J.S. y Potalsky, R.M. Iromaking Proc. Metall. Soc. AIME, 1990: 53-74. 